\title{
CMEARTICLE
}

\section{Clinics in diagnostic imaging (211)}

Eu Jo Martin Wong ${ }^{1}$, MB BCh BAO, Phey Ming Yeap ${ }^{1}$, MBChB, FRCR, Bak Siew Steven Wong ${ }^{1}$, MBChB, MMed

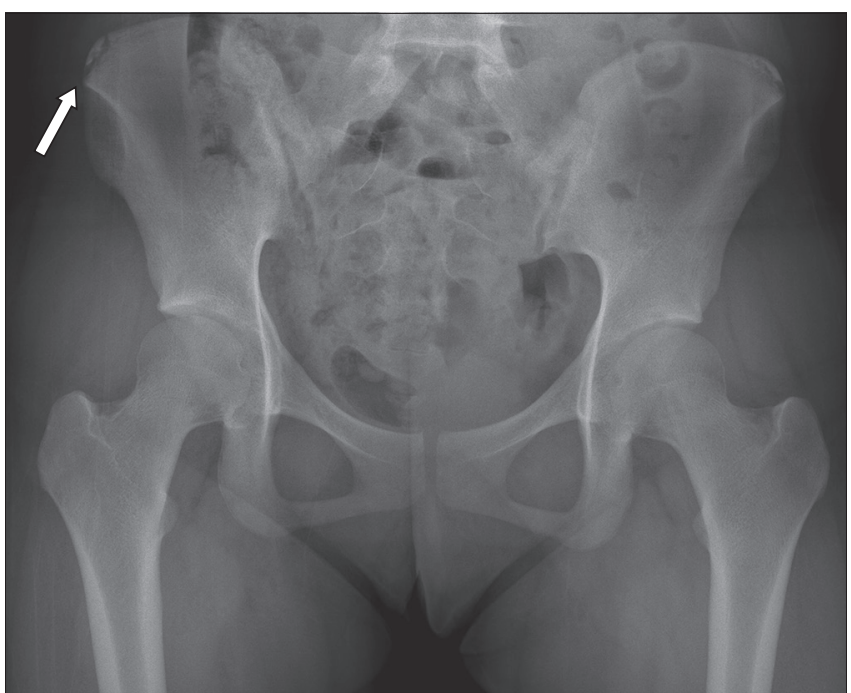

Fig. 1 Anteroposterior pelvic radiograph of a 14-year-old girl who complained of right hip pain following running in school.
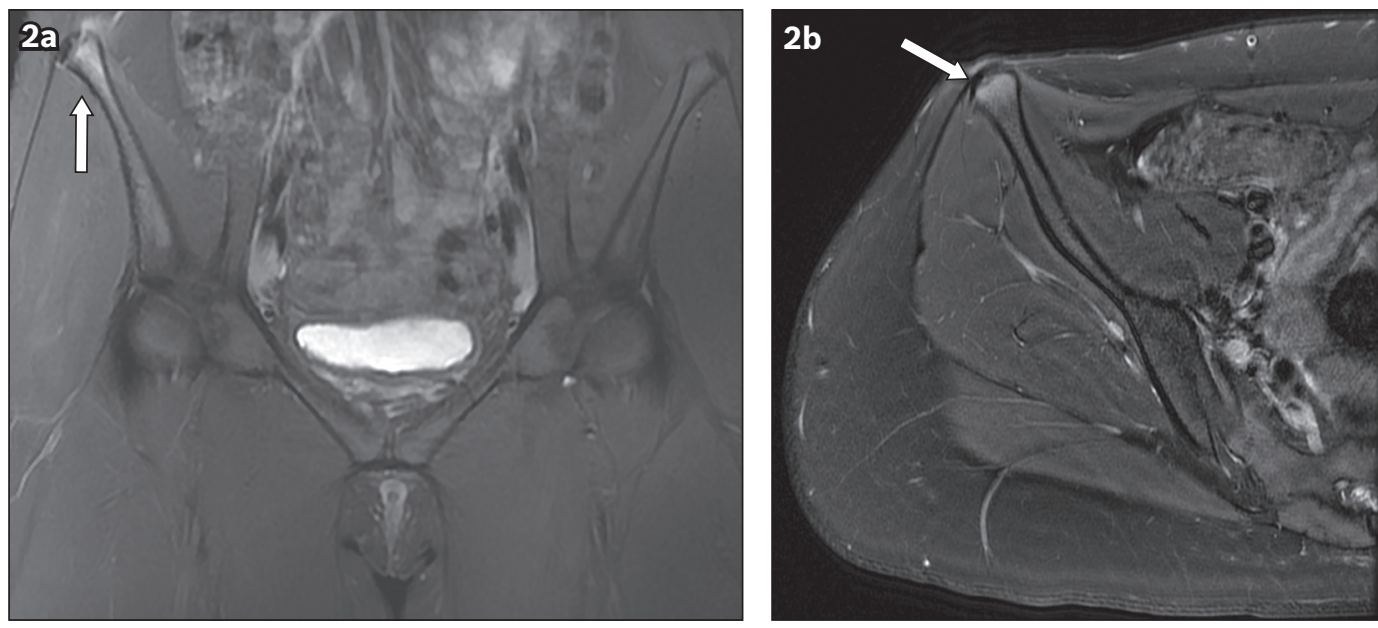

Fig. 2 (a) Coronal T2-W fat-suppressed (FS) MR image of the pelvis and (b) axial T2-W FS MR image of the right hemipelvis.

\section{CASE PRESENTATION}

A 14-year-old girl with no significant past medical history presented to our institution's sports medicine clinic for right groin pain following uphill running during a physical education class at school. Clinical examination revealed tenderness at the right anterior superior iliac spine (ASIS), anterior inferior iliac spine (AIIS) and pubic symphysis, with painful internal rotation.

Pelvic radiography (Fig. 1) and subsequent magnetic resonance (MR) imaging of the pelvis (Fig. 2) were performed to confirm the diagnosis. What do the radiograph and MR images show? What is the diagnosis? 

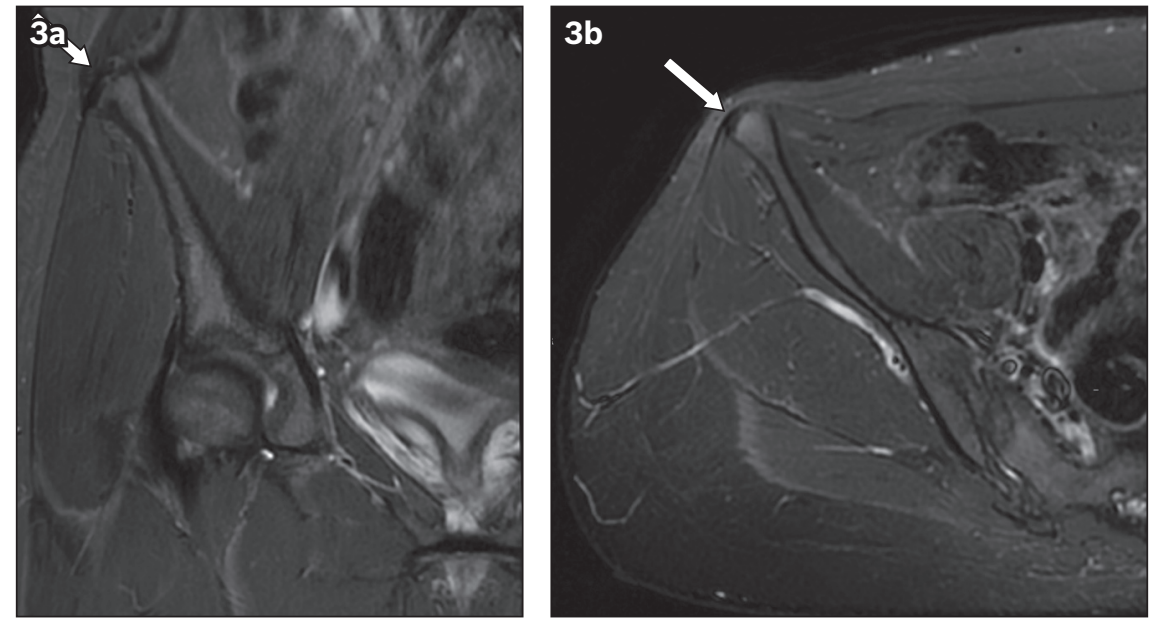

Fig. 3 (a) Coronal T2-W short tau inversion recovery (STIR) and (b) axial T2-W FS MR images of the right hemipelvis show resolution of the oedema within the abdominal muscles (arrows), with minimal bone marrow oedema in the iliac crest.

\section{IMAGE INTERPRETATION}

The anteroposterior pelvic radiograph (Fig. 1) shows asymmetric widening of the right iliac crest apophysis (arrow), suggesting right iliac apophysitis. No significant bony displacement of the apophysis was otherwise observed.

The coronal (Fig. 2a) and axial (Fig. 2b) fat-suppressed T2weighted (T2-W FS) MR images of the right hemipelvis show high signal in the anterior aspect of the right iliac crest (arrows), indicative of bone marrow oedema. Mild oedema is present in the adjacent lateral abdominal muscles - the external oblique, internal oblique and transverse abdominis muscles. No significant displacement of the iliac crest apophysis is noted.

\section{DIAGNOSIS}

Right iliac crest apophysitis.

\section{CLINICAL COURSE}

The patient was managed conservatively with analgesia and physiotherapy, and was advised to avoid running temporarily. Her symptoms subsequently improved, and she could gradually return to her baseline activities after 8-12 weeks.

Following physiotherapy and rehabilitation, the patient underwent repeat MR imaging of the pelvis (Fig. 3) three months after the initial MR imaging, prior to returning to more highly intense activities. The repeat MR image revealed resolution of the oedema within the abdominal muscles, with minimal residual bone marrow oedema in the iliac crest.

\section{DISCUSSION}

The bony pelvis develops from three primary ossification centres, namely the ilium, ischium and pubis, which fuse at the triradiate cartilage at approximately $12-14$ years of age. ${ }^{(1)}$ The apophyses are sites of attachment or origin of muscles that function as secondary growth centres, contributing to the shape or size of the bone but not to its length. ${ }^{(2)}$

The apophyses in the pelvis include the iliac crest, ischium, ASIS, AIIS, pubic tubercle, angle of pubis and ischial spine. The iliac crest apophysis is the attachment site for the lateral
Table I. Secondary ossification centres of the pelvis with median ages of appearance and closure. ${ }^{(4)}$

\begin{tabular}{|lll|}
\hline $\begin{array}{l}\text { Secondary ossification } \\
\text { centres }\end{array}$ & $\begin{array}{l}\text { Median age of } \\
\text { appearance }(\mathbf{y r})\end{array}$ & $\begin{array}{l}\text { Median age } \\
\text { of closure (yr) }\end{array}$ \\
\hline Iliac crest & 14.0 & 21.6 \\
\hline Ischial tuberosity & 14.0 & 20.6 \\
\hline Anterior superior iliac spine & 14.0 & 18.0 \\
\hline Anterior inferior iliac spine & 13.6 & 16.3 \\
\hline Greater trochanter & 4.1 & 16.8 \\
\hline Lesser trochanter & 10.9 & 14.1 \\
\hline
\end{tabular}

abdominal muscles (internal and external obliques, and transverse abdominis), gluteus muscles and tensor fascia latae.

The timing at which the pelvic secondary centres of ossification appear and fuse is further illustrated in Table I. Generally, the secondary ossification centres appear and fuse 1-2 years earlier in females, but individual variability exists. ${ }^{(3)}$ Knowledge about the sites of muscular insertion and the age of appearance and fusion of the apophyses is essential for differentiating normal-appearing apophyses from those in pathologic conditions.

The anatomy of the muscle-tendon-bone complex differs between adults and children. In children or adolescents, the unossified apophysis remains weaker than the attaching musculotendinous unit, rendering it vulnerable to both acute avulsion fractures ${ }^{(5)}$ and chronic stress injury or apophysitis. ${ }^{(6)}$ The anatomical sites of pelvic apophyseal injury with their muscular or tendinous attachments are illustrated in the pelvic radiograph in Fig. 4. The greater and lesser trochanters of the femur are the other two sites of apophyseal injury, which are included in a pelvic radiograph. Children and adolescents who actively participate in sports and other physical activities are at increased risk of developing apophyseal injuries. ${ }^{(6)}$ As more adolescents participate in athletic activities, they have a higher prevalence of such injuries. Apophyseal injuries in the pelvis are more common in boys, with the most commonly associated sports being football and track-and-field events, which commonly involve running or sprinting. ${ }^{(7)}$ Other athletic activities include gymnastics, athletics and tennis. The type of 


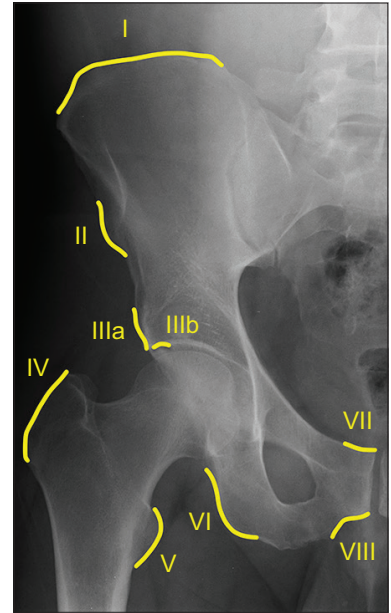

Fig. 4 Radiograph shows muscular origin/tendinous insertion sites: (I) iliac crest - abdominal muscles (internal and external obliques, and transverse abdominis), gluteus and tensor fascia latae; (II) anterior superior iliac spine - sartorius; (IIla) anterior inferior iliac spine - long head of rectus femoris; (IIIb) superior lateral acetabulum - reflected head of rectus femoris; (IV) greater trochanter - gluteus medius, gluteus minimus, gemellus and piriformis; (V) lesser trochanter - iliopsoas; (VI) ischial tuberosity - common hamstring tendon origin; (VII) pubic crest - rectus abdominis muscle; and (VIII) inferior pubic ramus - adductor muscles.

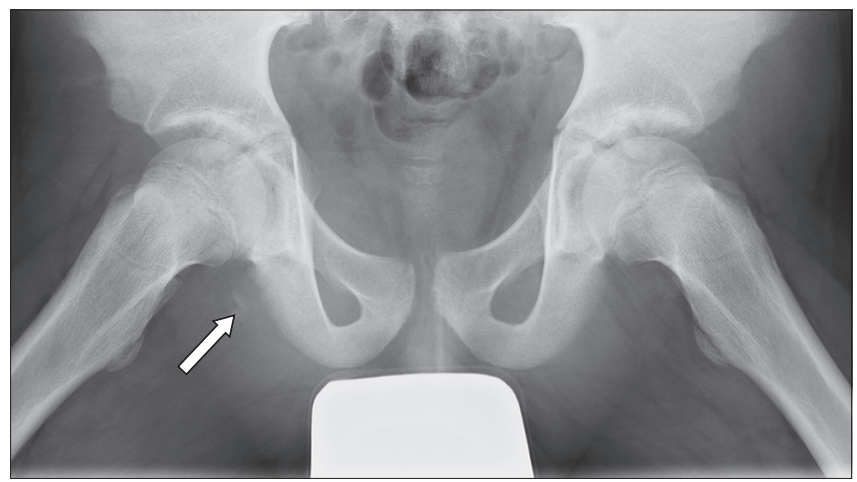

Fig. 6 Pelvic radiograph (performed with gonadal shielding) of a 14-yearold boy who was active in sports and presented with right hip pain shows an avulsion injury involving the right common hamstring muscle origin with a displaced fracture fragment of the right ischial tuberosity (arrow).

sporting activity and mechanism of injury will determine the site and severity of the apophyseal lesion. ${ }^{(8)}$

Acute injuries with avulsion often occur owing to excessive unbalanced muscular contraction, resulting in excruciating pain with loss of function and weakness. ${ }^{(8,9)}$ The mechanism for an acute iliac crest injury involves sudden contraction of the abdominal wall muscles during flexion and rotation of the torso. ${ }^{(9)}$ It has been hypothesised that the abrupt violent contraction of the external oblique muscle probably leads to avulsion of the iliac crest apophyses, and the unopposed antagonistic traction of the gluteus medius and tensor fascia latae leads to the lateral and inferior displacement of the avulsed fragment. ${ }^{(10)}$

A retrospective review of 228 cases by Schuett et al showed that the most common site of pelvic avulsion fractures was the AIIS (49\%), followed by the ASIS (30\%), ischial tuberosity (11\%) and iliac crest $(10 \%){ }^{(7)}$ Another series by Rossi and Dragoni, which included 203 cases, showed that the ischial tuberosity was the

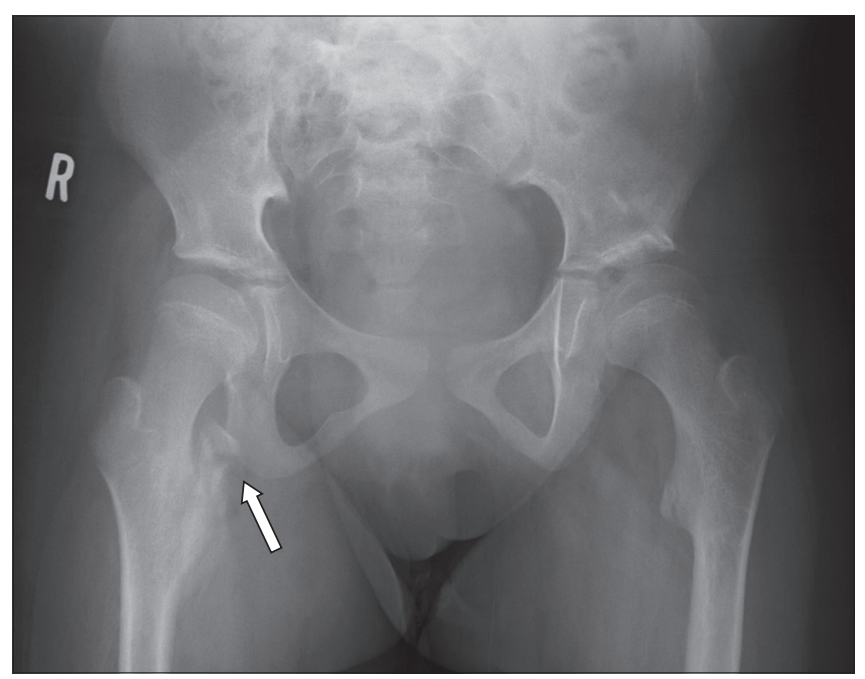

Fig. 5 Pelvic radiograph of an 11-year-old girl with recent sports injury at school shows an avulsion fracture of the right lesser trochanter with exuberant callus formation (arrow), which can be mistaken for an osseous tumour.

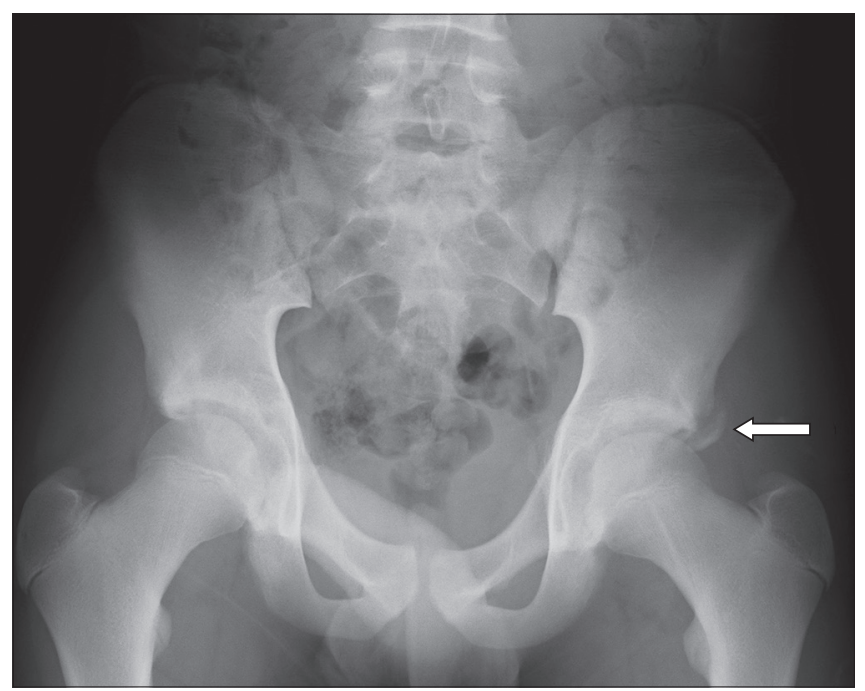

Fig. 7 Pelvic radiograph of a 16-year-old boy who presented with left hip pain following a football injury shows a left anterior inferior iliac spine avulsion fracture at the attachment site of the long head of the left rectus femoris (arrow).

most common site of avulsion fracture (53.6\%), followed by the AIIS (22.1\%), ASIS (19.2\%), superior corner of pubic symphysis $(3.4 \%)$ and iliac crest $(1.5 \%) .{ }^{(8)}$

Radiographs could identify up to $99 \%$ of all pelvic apophyseal avulsion fractures in Schuett et al's series, with 1\% requiring further evaluation with either computed tomography (CT) or MR imaging. As such, radiographs are often adequate for clinching a diagnosis, leaving more advanced imaging modalities for cases in which the radiographs are inconclusive and the diagnoses remain uncertain. ${ }^{(7)}$

Radiographs of an acute avulsion injury would confirm the presence of immature apophyses and may demonstrate a displaced fracture fragment adjacent to the site of muscular insertion. ${ }^{(11)}$ Figs. 5-8 illustrate the radiographic appearance of the different apophyseal avulsion fractures involving the pelvis and proximal femur. Fig. 9 is a radiograph of a skeletally mature 


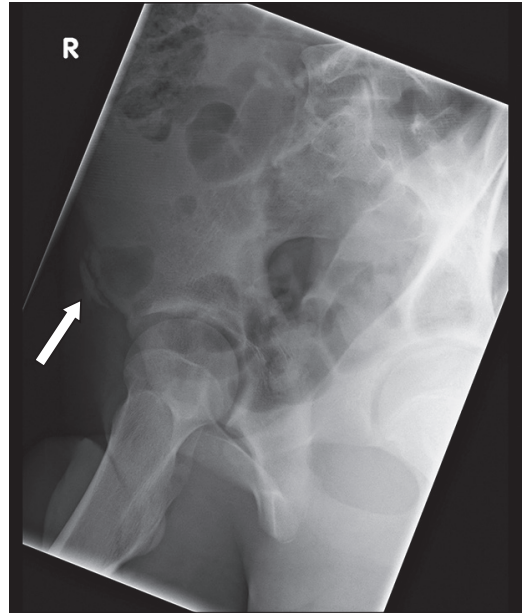

Fig. 8 Right hip radiograph of a 17-year-old boy with pain over the right iliac spine shows an avulsion fracture of the right anterior inferior iliac spine (arrow)

patient with an avulsion injury of the lesser trochanter; although this injury is exceedingly rare in the paediatric population, we would expect the radiographic appearance in a child to have a similar location and manifestation.

However, the initial radiographs can appear falsely normal in the setting of an acute apophyseal injury when there is no significant displacement of the apophyses or when the abnormality is bilateral and symmetrical. Radiographs may not demonstrate the injury if the apophysis has yet to ossify, generally in the setting of the younger paediatric population (below 14 years of age).

MR imaging is, therefore, the preferred imaging modality in diagnosing apophyseal injury, as it depicts the extent of injury including the displacement of apophysis and is useful in assessing the chronicity of the injury. In addition, the associated soft tissue injury, such as the integrity of the surrounding musculotendinous structures, can easily be assessed on MR imaging. ${ }^{(12)}$

Chronic or healing apophyseal avulsion fractures may appear as an abundant periosteal formation adjacent to the apophyses on radiographs. ${ }^{(13)}$ In some cases, the detached apophysis may lead to formation of irregular and exuberant callus, which may mimic a malignancy (Fig. 5), necessitating further evaluation with CT or MR imaging. ${ }^{(14)}$ Occasionally, it may be difficult to differentiate the imaging features between exuberant or hyperplastic callus formation from the tumour or an infection such as osteomyelitis; hence, knowing the overall clinical picture and clinical correlation is imperative. Some imaging features that would favour hyperplastic callus formation would include the presence of a fracture line, absence of medullary bone and soft tissue infiltration, absence of cortical bone destruction, and a homogeneous centre of callus without signs of central necrosis. ${ }^{(15)}$

Apophysitis or chronic injury is more insidious in onset and varies in severity, generally with no inciting history of trauma and typically arising from repetitive microtrauma or overuse. ${ }^{(14,16)}$ Iliac crest apophysitis is more prevalent in adolescents who participate in long-distance running. ${ }^{(7)}$ The initial radiograph may show asymmetry of the iliac crest apophyses, with separation of

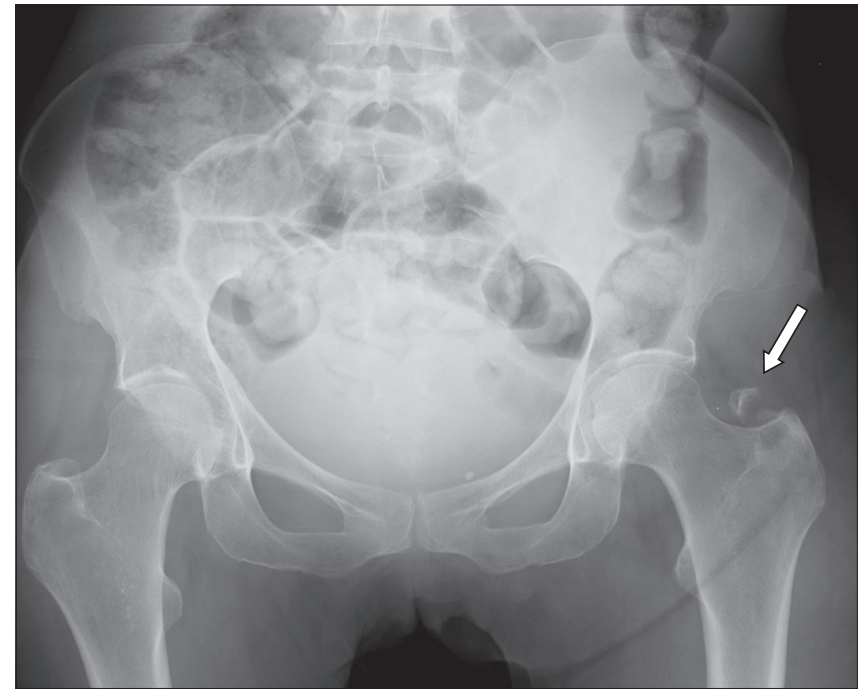

Fig. 9 Pelvic radiograph of a 25-year-old woman who presented with posttraumatic left hip pain shows left greater trochanter avulsion fracture at the gluteal tendon insertion site (arrow). This injury is very rarely encountered in the paediatric population. Although this is a radiograph of a skeletally mature patient, we would expect the radiographic image in a child to be similar in appearance.

the iliac crest from the iliac bone. ${ }^{(9)}$ However, some iliac crest apophysitis may be unperceivable on radiograph or even $\mathrm{CT}$, and MR imaging would be more sensitive in such cases. MR imaging features include physeal widening of 3-5 $\mathrm{mm}$ with increased signal intensity on water-sensitive sequences, bone marrow oedema within the iliac crest and oedema of the adjacent musculature. Displacement of the apophysis is generally absent in chronic injuries. ${ }^{(16)}$

Skeletal scintigraphy can be considered in certain cases. ${ }^{(13)}$ Generally, scintigraphy reveals non-specific findings, including asymmetric increased radiotracer uptake on the blood pool and delayed images. Scintigraphy is mainly used with atypical clinical presentations and when other imaging modalities are inconclusive. ${ }^{(17)}$ One potential disadvantage would be the risks of radiation to the patient.

Coincidentally, our index patient had an elder sister, a 15-yearold athlete, who presented with a two-month history of right hip pain. She participated in running in school and frequently performed sprinting during training. She also underwent MR imaging of the pelvis (Fig. 10), which confirmed the diagnosis of right iliac crest apophysitis. Pelvic avulsion fractures occurring in adolescent twins have been described in the literature, which raises the possibility of genetic predisposition in apophyseal injuries. ${ }^{(18)}$

Apophyseal injuries in adolescents are $\operatorname{rare}^{(7)}$ and are considered a challenge for the orthopaedic surgeon. In particular, iliac crest apophyseal injury accounts for only approximately $2 \%$ of pelvic fractures in adolescents. ${ }^{(8)}$ Hence, an accurate diagnosis is essential, especially for demanding patients and professional athletes, so that the patients are able to return to sports in optimal condition as soon as possible. Potential consequences of missed pelvic avulsion fractures include impingement and irritation of the sciatic or lateral femoral cutaneous nerves, especially during prolonged sitting, and chronic muscle weakness due to shortening 

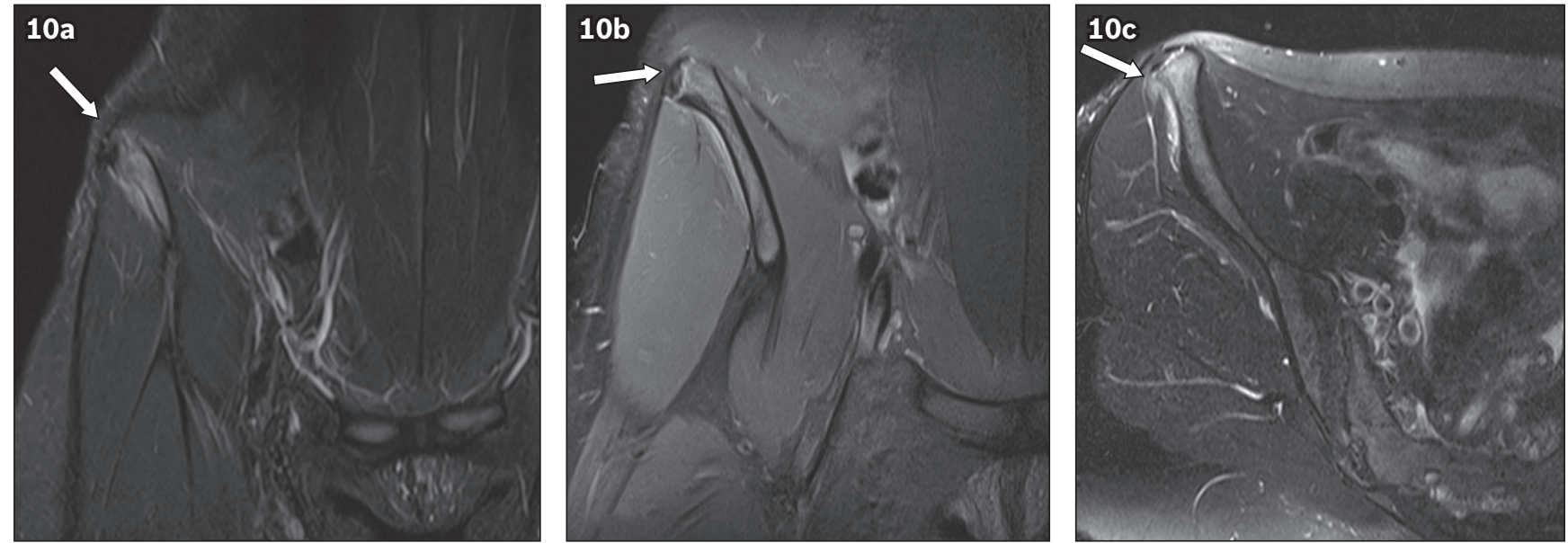

Fig. 10 (a) Coronal T2-W turbo inversion recovery magnitude; (b) coronal FS proton density; and (c) axial T2-W FS pelvic MR images of our index patient's 15-year-old elder sister, who presented with a two-month history of right hip pain following regular running, show high signal at the right iliac crest apophysis. There is mild widening of the iliac crest apophysis, with no significant displacement (arrows). Mild oedema is noted in the adjacent right gluteus and lateral abdominal muscles.

at the muscular origin, resulting in alterations in gait and inability to return to sports. ${ }^{(3)}$

Most cases of apophysitis and minimally displaced avulsion fractures involving the pelvic apophyses are treated with a period of altered weight bearing and slow return to activity, usually over a period of 6-12 weeks. $^{(3)}$ There is no well-established consensus for surgical management of these fractures in the literature. Generally, considerations for surgical management include the patient's physical demands, greater fracture fragments and displacement. ${ }^{(12)}$ A recent meta-analysis demonstrated that surgery has a better outcome than conservative management if the fracture fragment is larger than $15 \mathrm{~mm} .{ }^{(19)}$ Some authors favour surgical management if the fracture fragment is larger than $20 \mathrm{~mm}$ in its major axis and if the degree of dislocation exceeds $20 \mathrm{~mm} .{ }^{(20)}$ Other indications for surgery include chronic pain after conservative management or the presence of a large haematoma, which may compromise healing and require evacuation. Li et al showed that surgical management demonstrates good outcomes for patients, in that they are able to bear weight fully at two weeks and return to athletic activity at four weeks with no lower limb weakness. ${ }^{(21)}$

In summary, iliac crest apophysitis or avulsion is an uncommon injury. ${ }^{(7)}$ First-line imaging is usually by radiography, and other modalities such as CT or MR imaging can be considered if the initial diagnosis remains inconclusive and there is a high index of suspicion. Prompt and accurate diagnosis is imperative for the best outcome among young patients. Iliac crest apophysitis and minimally displaced avulsion fractures are usually treated conservatively with rest and physiotherapy, leading to gradual return to sports. A subgroup of patients with more significantly displaced fracture fragments or those with higher functional demands, such as athletes, may benefit from surgical management.

\section{REFERENCES}

1. Sankar WN, McCarthy JJ, Herman MJ. Pelvic and acetabular fractures. In: Waters PM, Skaggs DL, Flynn JM, eds. Rockwood and Wilkins' fracture in children. $9^{\text {th }}$ ed. Philadelphia, PA: Wolters Kluwer, 2020: 1347-406.
2. el-Khoury GY, Daniel WW, Kathol MH. Acute and chronic avulsive injuries. Radiol Clin North Am 1997; 35:747-66.

3. Schiller J, DeFroda S, Blood T. Lower extremity avulsion fractures in the pediatric and adolescent athlete. J Am Acad Orthop Surg 2017; 25:251-9.

4. Parvaresh KC, Upasani VV, Bomar JD, Pennock AT. Secondary ossification center appearance and closure in the pelvis and proximal femur. J Pediatr Orthop 2018; 38:418-23.

5. Singer G, Eberl R, Wegmann H, et al. Diagnosis and treatment of apophyseal injuries of the pelvis in adolescents. Semin Musculoskelet Radiol 2014; 18:498-504.

6. Zucker EJ, Lee EY, Restrepo R, Eisenberg RL. Hip disorders in children. AJR Am J Roentgenol 2013; 201:W776-96.

7. Schuett DJ, Bomar JD, Pennock AT. Pelvic apophyseal avulsion fractures: a retrospective review of 228 cases. J Pediatr Orthop 2015; 35:617-23.

8. Rossi F, Dragoni S. Acute avulsion fractures of the pelvis in adolescent competitive athletes: prevalence, location and sports distribution of 203 cases collected. Skeletal Radiol 2001; 30:127-31.

9. Steerman JG, Reeder MT, Udermann BE, Pettitt RW, Murray SR. Avulsion fracture of the iliac crest apophysis in a collegiate wrestler. Clin J Sport Med 2008; 18:102-3.

10. Coulier B. Acute avulsion of the iliac crest apophysis in an adolescent indoor soccer. J Belg Soc Radiol 2015; 99:20-4.

11. Vandervliet EJM, Vanhoenacker FM, Snoeckx A, et al. Sports-related acute and chronic avulsion injuries in children and adolescents with special emphasis on tennis. Br J Sports Med 2007; 41:827-31.

12. Calderazzi F, Nosenzo A, Galavotti C, et al. Apophyseal avulsion fractures of the pelvis. A review. Acta Biomed 2018; 89:470-6.

13. Connolly SA, Connolly LP, Jaramillo D. Imaging of sports injuries in children and adolescents. Radiol Clin North Am 2001; 39:773-90.

14. Raissaki M, Apostolaki E, Karantanas AH. Imaging of sports injuries in children and adolescents. Eur J Radiol 2007; 62:86-96.

15. Dobrocky I, Seidl G, Grill F. MRI and CT features of hyperplastic callus in osteogenesis imperfecta tarda. Eur Radiol 1999; 9:665-8.

16. Hébert KJ, Laor T, Divine JG, Emery KH, Wall EJ. MRI appearance of chronic stress injury of the iliac crest apophysis in adolescent athletes. AJR Am J Roentgenol 2008; 190:1487-91.

17. Arnaiz J, Piedra T, de Lucas EM, et al. Imaging findings of lower limb apophysitis. AJR Am J Roentgenol 2011; 196:W316-25.

18. Reina N, Accadbled F, de Gauzy JS. Anterior inferior iliac spine avulsion fracture: a case report in soccer playing adolescent twins. J Pediatr Orthop B 2010; 19:158-60.

19. Eberbach $\mathrm{H}$, Hohloch L, Feucht MJ, et al. Operative versus conservative treatment of apophyseal avulsion fractures of the pelvis in the adolescents: a systematical review with meta-analysis of clinical outcome and return to sports. BMC Musculoskelet Disord 2017; 18:162.

20. Pogliacomi F, Calderazzi F, Paterlini M, Ceccarelli F. Surgical treatment of anterior iliac spines fractures: our experience. Acta Biomed 2014; 85 Suppl 2:52-8.

21. Li X, Xu S, Lin X, Wang Q, Pan J. Results of operative treatment of avulsion fractures of the iliac crest apophysis in adolescents. Injury 2014; 45:721-4 


\section{SINGAPORE MEDICAL COUNCIL CATEGORY 3B CME PROGRAMME} (Code SMJ 202109B)

Question 1. Regarding apophyses:

True False

(a) They contribute to the shape and size of the bone but not its length.

(b) They generally appear and fuse earlier in males.

(c) Sedentary patients are at higher risk of developing apophyseal injury.

(d) The iliac crest is the most common site of avulsion fracture.

Question 2. Regarding muscular attachments in the pelvis and proximal femur:

(a) The iliopsoas attaches to the greater trochanter.

(b) The rectus femoris attaches to the anterior superior iliac spine.

(c) The abdominal wall muscles attach to the iliac crest.

(d) The gluteus medius and minimus attach to the greater trochanter.

Question 3. Regarding imaging for apophyseal injuries:

(a) Magnetic resonance (MR) imaging or computed tomography (CT) is always required for further evaluation.

(b) MR imaging is more sensitive than CT for detection of apophyseal injury.

(c) MR imaging is able to depict the extent of apophyseal injury and associated soft tissue injury.

(d) Skeletal scintigraphy is routinely performed for evaluation of apophyseal injury.

Question 4. Regarding imaging for apophyseal injuries:

(a) Appearances of chronic or healing apophyseal avulsion fractures may rarely mimic an aggressive lesion.

(b) Radiographs may not demonstrate the injury in the younger paediatric population.

(c) MR imaging findings include bone marrow oedema and oedema of the adjacent musculature on watersensitive sequences.

(d) Increased radiotracer uptake on scintigraphy is specific for apophyseal injury.

Question 5. Regarding treatment options:

(a) Surgical treatment is recommended for all patients.

(b) Surgical treatment is favoured for larger fracture fragments and larger degrees of displacement.

(c) Patients with greater physical demand may benefit more from surgery.

(d) Chronic pain and alterations in gait are known sequelae if not treated appropriately.

$\begin{array}{ll}\square & \square \\ \square & \square \\ \square & \square \\ \square & \square\end{array}$

$\square$

\section{Doctor's particulars:}

Name in full:

Specialty:
MCR no.:

Email:

\section{SUBMISSION INSTRUCTIONS:}

Visit the SMJ website: http://www.smj.org.sg/current-issue and select the appropriate quiz. You will be redirected to the SMA login page.

For SMA member: (1) Log in with your username and password (if you do not know your password, please click on 'Forgot your password?'). (2) Select your answers for each quiz and click 'Submit'.

For non-SMA member: (1) Create an SMJ CME account, or log in with your SMJ CME username and password (for returning users). (2) Make payment of SGD 21.40 (inclusive of $7 \%$ GST) via PayPal to access this month's quizzes. (3) Select your answers for each quiz and click 'Submit'.

\section{RESULTS:}

(1) Answers will be published online in the SMJ November 2021 issue. (2) The MCR numbers of successful candidates will be posted online at the SMJ website by 30 November 2021. (3) Passing mark is $60 \%$. No mark will be deducted for incorrect answers. (4) The SMJ editorial office will submit the list of successful candidates to the Singapore Medical Council. (5) One CME point is awarded for successful candidates. (6) SMC credits CME points according to the month of publication of the CME article (i.e. points awarded for a quiz published in the September 2021 issue will be credited for the month of September 2021, even if the deadline is in November 2021).

Deadline for submission (September 2021 SMJ 3B CME programme): 12 noon, 23 November 2021. 